\title{
FACULTY OF SPORTS SCIENCES STUDENTS' LEARNING LEVELS OF THE PRACTICAL COURSES DURING THE PANDEMIC PERIOD
}

\author{
Mustafa Kaya ${ }^{1 i}$, \\ Alperen Çırçır², \\ Alper Eyinç3, \\ Mehmet Kargün, \\ Recep Bozdemir ${ }^{5}$ \\ ${ }^{1}$ Sivas Cumhuriyet University, \\ Faculty of Sport Sciences, \\ Coaching Training Department, \\ Turkey \\ ${ }^{2}$ Sivas Cumhuriyet University, \\ Health Sciences Institute, \\ Turkey \\ ${ }^{3}$ Sivas Cumhuriyet University, \\ Yildizeli Vocational School, \\ Turkey \\ ${ }^{4}$ Tokat Gaziosmanpaşa University, \\ Sport Sciences, \\ Sports Management Department, \\ Turkey \\ ${ }^{5}$ Tokat Gaziosmanpaşa University, \\ Sport Sciences, \\ Coaching Training Department, \\ Turkey
}

\begin{abstract}
:
This research was carried out to analyze the attitude and learning levels of the Faculty of Sport Sciences students related to the practical courses lectured in distance education due to the isolation days. This research was taught to examine the attitudes of the sports sciences faculty coaching education department towards the applied lessons taught in distance education from the meal isolation days. The sample group of the study included totally 460 students including 233 females and 227 males studying at the Sport Sciences Faculties of universities in 2020-2021 Academic Year. The demographic properties of the students participating into the study such as the variables of gender, grade and department were recorded. Furthermore, 4 questionnaire questions with "yes" or "no" answers prepared by the researcher and a 10-item questionnaire for questioning the
\end{abstract}

i Correspondence email: mustafakaya58@cumhuriyet.edu.tr 
students' attitudes towards learning in practical courses lectured in distance education were created. The statistical values were calculated in SPSS program. The arithmetic average and standard deviation values of the obtained data were calculated. The ChiSquare Test (x2) was performed to determine whether the department and grade variables had a significant effect upon the answers given to the attitude questions. In conclusion, it was determined that the practical courses lectured in distance education in Faculty of Sports Sciences negatively affected the learning levels of students during the pandemic caused by the Covid-19 virus.

Keywords: Covid-19 pandemic, distance practical education, university students

\section{Introduction}

On December 31, 2019, a new case that started with fever of unknown etiology, dry cough dyspnea, and pneumonia resulted in death was reported in Wuhan, China's Hubei province (Dong et al., 2020, Jiménez et al., 2020, Palacios Cruz et al., 2021, Aktaş et al., 2020). The virus was reported to be infected from person to person by droplets or by contact of the respiratory secretion materials of the sick person with the mouth, nose, eyes and mucous membranes of healthy people ( $\mathrm{Li}$ et al., 2020). As of February 17, 2020, the pandemic has spread to every province of China and 27 different countries in the world with more than 70,000 confirmed cases. It was declared as a pandemic on 11 March 2020 due to the very rapid spread of the disease and its high infection rate (Dong et al., 2020). The countries that have been negatively affected from the increase in the rate of cases and deaths every day in the global pandemic have had to take measures in many areas affecting and reorganizing the social life. The measures decided to minimize the infection rate of the rapidly spreading corona virus and the adverse effects of the mortality rate have been taken in our lives without loss of any time (Çelik and Yenal, 2020).

The first Covid-19 case in Turkey was diagnosed in March 2020, and some precautions were necessarily taken in educational activities in order to prevent the spread of the virus (Afşar and Büyükdoğan, 2020). First of all, education was suspended for three weeks in all primary, secondary and higher education institutions including preschool education institutions as of March 16, 2020. Subsequent to the increase at the number of cases, all education levels were decided to be maintained in distance education method until the end of 2019-2020 spring semester. At the meeting held on March 13, Higher Education Council decided to suspend education in the universities in Turkey for 3 weeks as of March 16, 2021, and subsequently, the educational activities at universities that had distance education infrastructure was decided to be executed distance as of March 23, 2020 (Şen and Kızılcalığlu, 2020, YÖK-Higher Education Council, 2020). So that, at the same time millions of students were simultaneously started to be lectured in distance education courses in Turkey.

Distance education is defined as a form of education that is not possible to be carried on in traditional teaching techniques enabling students and teachers to be 
independent from each other in different places with a more flexible but a certain standard, being low cost, allowing continuity in learning, being more practical and offering more diversity within the scope of measurement and evaluation (Demir, 2014). Today's distance education processes cannot be fully an alternative to face-to-face implementations yet (Tuncer and Bahadır, 2017). In this sense, the dimension of interaction provided in face-to-face education is possible to be expressed as the most important deficiency for distance education. The distance education system was generally used in theoretical courses in the period before the pandemic. For this reason, difficulties have arisen in administering the distance education model in departments with practical course curriculum whereas it can be easily administered in theoretical courses after the pandemic. While the distance education process is possible to be considered as efficient for theoretical courses, appropriate curricula and content for practical courses is noticed to be developed.

In education curricula of the Faculty of Sport Sciences and School of Physical Education and Sports, the courses have been conducted in two ways as theoretical and practical. Some of these courses are only theoretical, some are theoretical and practical, and some others are only taught in practice. Administering theoretical and practical courses together is extremely important in terms of putting the learned knowledge into practice, using the body effectively, comprehending and performing individual-team and field courses. In the current curriculum, the Physical Education and Sports Teaching Department's total course hours in 8 semesters is 171 including 42 hours $(24.5 \%)$ as practice; the total course hours of the Coaching Education Department is 171 including 56 hours $(31.8 \%)$ as practice; the total course hours of the Department of Sports Management is 174, and 26 hours (14.9\%) of these are practice and the total course hours of the Recreation Department are 206, 42 hours (20.3\%) of these are practice courses. According to this rate, the practice courses which create a large part of the general course hour base are extremely valuable in terms of lecturing the courses in Faculties of Sport Sciences. Moreover, especially the practical courses enable the students acquire experiences in terms of using the demonstration method in their future professional lives with their students and teaching the acts of their course. In this context, it is considered that administering practical courses face to face is possible to be more beneficial for students studying in sports sciences. It is suggested that the not administering face-toface education during the pandemic period has had negative effects upon the educational processes of departments that have practice courses such as Faculty of Sports Sciences and School of Physical Education and Sports. The distance education system's quality of providing theoretical subjects reveals some inadequacies for students of the departments with practical courses. Due to the limited number of studies on the subject, to what extent these inadequacies will affect students is not known well. In this sense, it is very important to investigate the subject. 
This research was carried out to determine the effects of the practice courses lectured in distance education during the pandemic period on learning levels of the students studying at Faculty of Sports Sciences of the universities.

\section{Method}

This research was designed using the screening model. The screening model was used for studies carried out on larger samples investigating the properties such as interests, skills, attitudes and views of the participants upon a subject or event (Büyüköztürk et al., 2008). The random method was used in sample selection (Baştürk and Taştepe, 2013). The sample group of the study totally included 460 university volunteer students including 233 females (50.7\%) and 227 males (49.3\%) studying at the Faculty of Sport Sciences of various universities in the Fall Semester of the 2020-2021 Academic Year. The questionnaire form was performed to the students online, and the necessary explanations were given in the questionnaire before starting the study.

\subsection{Data Collection Tools}

\subsubsection{Personal Information Form}

In the study, the personal information form created by the researchers was used to determine the demographic properties of the participants such as age, gender, the department they studied, the university they studied, and the practical courses they took with distance education during the pandemic period.

\subsubsection{Attitude Questionnaire}

The questionnaire form prepared by the researchers using the examples in the literature was used in order to determine the participants' attitudes towards distance education courses and exams due to the isolation days caused by Covid-19 virus. The questionnaire form included 10 items to be answered as "I agree", "Neither agree nor disagree" and "I disagree". The items asked in the questionnaire were listed below.

\subsection{Data Analysis}

SPSS 25 statistical analysis software was used to analyze the data. These data were expressed in frequency and percentage. In addition, the Chi-Square Test (x2) was performed for determining whether the variables of department and grade had a significant effect upon the answers given to the attitude questions.

\subsection{Ethical Aspect of the Research}

Ethical permission was obtained as result of the decision number 20 taken in 02.04.2021 dated and E-60263016-050.06.04-29083 numbered Sivas Cumhuriyet University Scientific Research and Publication Ethics Social and Human Sciences Committee meeting. 


\section{Findings}

Table 1: Demographic Properties of the Students and Their Views on Distance Education

\begin{tabular}{|c|c|c|c|}
\hline \multirow{3}{*}{ Gender } & & $\mathbf{N}$ & $\%$ \\
\hline & Male & 227 & 49,3 \\
\hline & Female & 233 & 50,7 \\
\hline \multirow[t]{4}{*}{ Department } & $\begin{array}{l}\text { Department of Physical } \\
\text { Education and Sports Teaching }\end{array}$ & 187 & 41,3 \\
\hline & $\begin{array}{l}\text { Department of Coaching } \\
\text { Training }\end{array}$ & 111 & 24,5 \\
\hline & $\begin{array}{l}\text { Department of Sports } \\
\text { Management }\end{array}$ & 135 & 29,8 \\
\hline & Department of Recreation & 20 & 4,4 \\
\hline \multirow[t]{4}{*}{ Grade } & $1^{\text {st }}$ Grade & 115 & 25,4 \\
\hline & $2^{\text {nd }}$ Grade & 71 & 15,7 \\
\hline & $3^{\text {rd }}$ Grade & 91 & 20,1 \\
\hline & $4^{\text {th }}$ Grade & 176 & 38,9 \\
\hline \multirow{2}{*}{$\begin{array}{l}\text { Would you like distance education process to } \\
\text { continue? }\end{array}$} & Yes & 116 & 25,1 \\
\hline & No & 346 & 74,9 \\
\hline \multirow{2}{*}{$\begin{array}{l}\text { Do you consider the current distance education } \\
\text { system in your university efficacious? }\end{array}$} & Yes & 211 & 45,7 \\
\hline & No & 251 & 54,3 \\
\hline \multirow{2}{*}{$\begin{array}{l}\text { Can you connect to the distance education system at } \\
\text { your university without experiencing any problems? }\end{array}$} & Yes & 271 & 58,5 \\
\hline & No & 192 & 41,5 \\
\hline \multirow{2}{*}{$\begin{array}{l}\text { Do you benefit from the distance education system } \\
\text { at the university you are studying? }\end{array}$} & Yes & 273 & 59,3 \\
\hline & No & 187 & 40,7 \\
\hline
\end{tabular}

Table 2: The Attitude Results of Students against Practical Courses Lectured During Isolation Days Caused by Covid-19 Virus

\begin{tabular}{|l|c|c|c|c|c|c|}
\hline \multirow{2}{*}{} & \multicolumn{2}{|c|}{$\begin{array}{c}\text { I } \\
\text { agree }\end{array}$} & \multicolumn{2}{c|}{$\begin{array}{c}\text { Neither Agree } \\
\text { nor Disagree }\end{array}$} & \multicolumn{2}{|c|}{$\begin{array}{c}\text { I } \\
\text { Disagree }\end{array}$} \\
\cline { 2 - 7 } & $\mathbf{n}$ & $\mathbf{\%}$ & $\mathbf{n}$ & $\mathbf{\%}$ & $\mathbf{n}$ & $\mathbf{\%}$ \\
\hline $\begin{array}{l}\text { I think the practical courses lectured in distance education } \\
\text { process will create a deficiency and negativity in my } \\
\text { education life in the next semester or normal life. }\end{array}$ & 317 & 68,3 & 84 & 18,1 & 63 & 13,6 \\
\hline $\begin{array}{l}\text { I do not consider the opportunities offered by our school for } \\
\text { practical courses in distance education process as } \\
\text { efficacious. }\end{array}$ & 254 & 54,7 & 137 & 29,5 & 73 & 15,7 \\
\hline $\begin{array}{l}\text { I do not think the education before the pandemic period and } \\
\text { the education during the distance education period have } \\
\text { created a negative difference to my learning level. }\end{array}$ & 165 & 35,6 & 146 & 31,5 & 153 & 33,0 \\
\hline $\begin{array}{l}\text { I do not think the courses lectured in distance education for } \\
\text { the Faculty of Sport Sciences students during the pandemic } \\
\text { period are suitable for our department. }\end{array}$ & 300 & 64,7 & 97 & 20,9 & 67 & 14,4 \\
\hline $\begin{array}{l}\text { I do not think the theoretical expression of my practical } \\
\text { courses in distance education process contributes upon my } \\
\text { learning level. }\end{array}$ & 293 & 63,1 & 100 & 21,6 & 71 & 15,3 \\
\hline
\end{tabular}




\begin{tabular}{|l|c|c|c|c|c|c|}
\hline \hline $\begin{array}{l}\text { I would like the measurement and evaluation of my } \\
\text { practical courses to be fulfilled online after the pandemic } \\
\text { period is over. }\end{array}$ & 149 & 32,1 & 145 & 31,3 & 170 & 36,6 \\
\hline $\begin{array}{l}\text { I would like my practical courses to be lectured by visual or } \\
\text { live presentations instead of theoretical expression in } \\
\text { distance education process. }\end{array}$ & 317 & 68,3 & 100 & 21,6 & 47 & 10,1 \\
\hline $\begin{array}{l}\text { I would like to take my practical courses face to face if there } \\
\text { were opportunities during the pandemic period. }\end{array}$ & 346 & 74,6 & 74 & 15,9 & 44 & 9,5 \\
\hline $\begin{array}{l}\text { I do not believe that I will be able to put my theoretical } \\
\text { practical courses into practice in distance education. }\end{array}$ & 224 & 48,3 & 173 & 37,3 & 67 & 14,4 \\
\hline $\begin{array}{l}\text { I do not consider my learning level in practical courses } \\
\text { adequate in distance education process. }\end{array}$ & 298 & 64,2 & 106 & 22,8 & 60 & 12,9 \\
\hline
\end{tabular}

When the preferences of the students participating into the study in a 3-point Likert-type scale were analyzed in Table 2, it was noticed that whereas the rate of "I Agree" was high $(\% 36,6)$ only in the item of "I would like the measurement and evaluation of my practical courses to be fulfilled online after the pandemic period is over," the rate of "I agree" was high for all the remaining items.

Table 3: The Chi-Square Test Results According to the Variable of Grade in Attitude Levels of the Students towards the Practice Courses Lectured in Distance Education during the Pandemic Period Caused by the Covid-19 Virus

\begin{tabular}{|c|c|c|c|c|c|c|c|c|c|c|}
\hline & & \multicolumn{2}{|c|}{$\begin{array}{c}1^{\text {st }} \\
\text { Grade }\end{array}$} & \multicolumn{2}{|c|}{$\begin{array}{c}2^{\text {nd }} \\
\text { Grade }\end{array}$} & \multicolumn{2}{|c|}{$\begin{array}{c}3^{\text {rd }} \\
\text { Grade }\end{array}$} & \multicolumn{2}{|c|}{$\begin{array}{c}4^{\text {th }} \\
\text { Grade }\end{array}$} & \multirow{2}{*}{$\begin{array}{c}\begin{array}{c}\text { Pearson } \\
\text { Chi- } \\
\text { Square }\end{array} \\
p \\
\end{array}$} \\
\hline & & $\mathbf{n}$ & $\%$ & $\mathbf{n}$ & $\%$ & $\mathbf{n}$ & $\%$ & $\mathbf{n}$ & $\%$ & \\
\hline \multirow{3}{*}{$\begin{array}{l}\text { I think the practical courses } \\
\text { lectured in distance education } \\
\text { process will create a deficiency and } \\
\text { negativity in my education life in } \\
\text { the next semester or normal life. }\end{array}$} & I Agree & 84 & 73,0 & 46 & 64,8 & 64 & 70,3 & 115 & 65,3 & \multirow{3}{*}{ 118 } \\
\hline & $\begin{array}{l}\text { Neither } \\
\text { Agree nor } \\
\text { Disagree }\end{array}$ & 24 & 20,9 & 14 & 19,7 & 15 & 16,5 & 28 & 15,9 & \\
\hline & I Disagree & 7 & 6,1 & 11 & 15,5 & 12 & 13,2 & 33 & 18,8 & \\
\hline \multirow{3}{*}{$\begin{array}{l}\text { I do not consider the opportunities } \\
\text { offered by our school for practical } \\
\text { courses in distance education } \\
\text { process as efficacious. }\end{array}$} & I Agree & 56 & 48,7 & 40 & 56,3 & 60 & 65,9 & 94 & 53,4 & \multirow{3}{*}{,094 } \\
\hline & $\begin{array}{l}\text { Neither } \\
\text { Agree nor } \\
\text { Disagree }\end{array}$ & 42 & 36,5 & 23 & 32,4 & 17 & 18,7 & 49 & 27,8 & \\
\hline & I Disagree & 17 & 14,8 & 8 & 11,3 & 14 & 15,4 & 33 & 18,8 & \\
\hline \multirow{3}{*}{$\begin{array}{l}\text { I do not think the education before } \\
\text { the pandemic period and the } \\
\text { education during the distance } \\
\text { education period have created a } \\
\text { negative difference to my learning } \\
\text { level. }\end{array}$} & I Agree & 38 & 33,0 & 26 & 36,6 & 33 & 36,3 & 65 & 36,9 & \multirow{3}{*}{,720 } \\
\hline & $\begin{array}{l}\text { Neither } \\
\text { Agree nor } \\
\text { Disagree }\end{array}$ & 44 & 38,3 & 23 & 32,4 & 25 & 27,5 & 52 & 29,5 & \\
\hline & I Disagree & 33 & 28,7 & 22 & 31,0 & 33 & 36,3 & 59 & 33,5 & \\
\hline \multirow{4}{*}{$\begin{array}{l}\text { I do not think the courses lectured } \\
\text { in distance education for the } \\
\text { Faculty of Sport Sciences students } \\
\text { during the pandemic period are } \\
\text { suitable for our department. }\end{array}$} & I Agree & 77 & 67,0 & 50 & 70,4 & 59 & 64,8 & 105 & 59,7 & \multirow{3}{*}{,620 } \\
\hline & $\begin{array}{l}\text { Neither } \\
\text { Agree nor } \\
\text { Disagree }\end{array}$ & 22 & 19,1 & 10 & 14,1 & 19 & 20,9 & 44 & 25,0 & \\
\hline & I Disagree & 16 & 13,9 & 11 & 15,5 & 13 & 14,3 & 27 & 15,3 & \\
\hline & I Agree & 76 & 66,1 & 45 & 63,4 & 59 & 64,8 & 106 & 60,2 & ,594 \\
\hline
\end{tabular}


Mustafa Kaya, Alperen Çırçır, Alper Eyinç, Mehmet Kargün, Recep Bozdemir

FACULTY OF SPORTS SCIENCES STUDENTS' LEARNING LEVELS OF

THE PRACTICAL COURSES DURING THE PANDEMIC PERIOD

\begin{tabular}{|c|c|c|c|c|c|c|c|c|c|c|}
\hline \multirow{2}{*}{$\begin{array}{l}\text { I do not think the theoretical } \\
\text { expression of my practical courses } \\
\text { in distance education process } \\
\text { contributes upon my learning } \\
\text { level. }\end{array}$} & $\begin{array}{l}\text { Neither } \\
\text { Agree nor } \\
\text { Disagree } \\
\end{array}$ & 26 & 22,6 & 13 & 18,3 & 15 & 16,5 & 42 & 23,9 & \\
\hline & I Disagree & 13 & 11,3 & 13 & 18,3 & 17 & 18,7 & 28 & 15,9 & \\
\hline \multirow{3}{*}{$\begin{array}{l}\text { I would like the measurement and } \\
\text { evaluation of my practical courses } \\
\text { to be fulfilled online after the } \\
\text { pandemic period is over. }\end{array}$} & I Agree & 34 & 29,6 & 25 & 35,2 & 30 & 33,0 & 55 & 31,3 & \multirow{3}{*}{,965 } \\
\hline & $\begin{array}{l}\text { Neither } \\
\text { Agree nor } \\
\text { Disagree }\end{array}$ & 38 & 33,0 & 21 & 29,6 & 25 & 27,5 & 57 & 32,4 & \\
\hline & I Disagree & 43 & 37,4 & 25 & 35,2 & 36 & 39,6 & 64 & 36,4 & \\
\hline \multirow{3}{*}{$\begin{array}{l}\text { I would like my practical courses } \\
\text { to be lectured by visual or live } \\
\text { presentations instead of theoretical } \\
\text { expression in distance education } \\
\text { process. }\end{array}$} & I Agree & 88 & 76,5 & 50 & 70,4 & 61 & 67,0 & 110 & 62,5 & \multirow{3}{*}{,075 } \\
\hline & $\begin{array}{l}\text { Neither } \\
\text { Agree nor } \\
\text { Disagree } \\
\end{array}$ & 22 & 19,1 & 16 & 22,5 & 20 & 22,0 & 39 & 22,2 & \\
\hline & I Disagree & 5 & 4,3 & 5 & 7,0 & 10 & 11,0 & 27 & 15,3 & \\
\hline \multirow{3}{*}{$\begin{array}{l}\text { I would like to take my practical } \\
\text { courses face to face if there were } \\
\text { opportunities during the pandemic } \\
\text { period. }\end{array}$} & I Agree & 97 & 84,3 & 56 & 78,9 & 70 & 76,9 & 114 & 64,8 & \multirow{3}{*}{, $002^{* *}$} \\
\hline & $\begin{array}{l}\text { Neither } \\
\text { Agree nor } \\
\text { Disagree }\end{array}$ & 12 & 10,4 & 5 & 7,0 & 13 & 14,3 & 42 & 23,9 & \\
\hline & I Disagree & 6 & 5,2 & 10 & 14,1 & 8 & 8,8 & 20 & 11,4 & \\
\hline \multirow{3}{*}{$\begin{array}{l}\text { I do not believe that I will be able } \\
\text { to put my theoretical practical } \\
\text { courses into practice in distance } \\
\text { education. }\end{array}$} & I Agree & 56 & 48,7 & 36 & 50,7 & 50 & 54,9 & 77 & 43,8 & \multirow{3}{*}{, $013^{*}$} \\
\hline & $\begin{array}{l}\text { Neither } \\
\text { Agree nor } \\
\text { Disagree } \\
\end{array}$ & 50 & 43,5 & 27 & 38,0 & 21 & 23,1 & 70 & 39,8 & \\
\hline & I Disagree & 9 & 7,8 & 8 & 11,3 & 20 & 22,0 & 29 & 16,5 & \\
\hline \multirow{3}{*}{$\begin{array}{l}\text { I do not consider my learning level } \\
\text { in practical courses adequate in } \\
\text { distance education process. }\end{array}$} & I Agree & 77 & 67,0 & 47 & 66,2 & 65 & 71,4 & 103 & 58,5 & \multirow{3}{*}{,448 } \\
\hline & $\begin{array}{l}\text { Neither } \\
\text { Agree nor } \\
\text { Disagree } \\
\end{array}$ & 25 & 21,7 & 14 & 19,7 & 16 & 17,6 & 47 & 26,7 & \\
\hline & I Disagree & 13 & 11,3 & 10 & 14,1 & 10 & 11,0 & 26 & 14,8 & \\
\hline
\end{tabular}

${ }^{*} \mathrm{p}<0,05,{ }^{* *} \mathrm{p}<0,01$

When the Attitude Levels of the students participating into the study towards the Practice Courses Lectured in Distance Education were analyzed, it was determined that there was a statistically significant difference $(p<0.05)$ according to the variable of grade in the answers given to the item of "I would like to take my practical courses face to face if there were opportunities during the pandemic period," and to the item of "I do not believe that I will be able to put my theoretical practical courses into practice in distance education" and no significant difference was found in the other items $(p>0.05)$. 
Table 4: The Chi-Square Test Results According to the Variable of Department in Attitude Levels of the Students towards the Practice Courses Lectured in Distance Education during the Pandemic Period Caused by the Covid-19 Virus

\begin{tabular}{|c|c|c|c|c|c|c|c|c|c|c|}
\hline & & \multicolumn{2}{|c|}{$\begin{array}{l}\text { Department } \\
\text { of Physical } \\
\text { Education } \\
\text { and Sports }\end{array}$} & \multicolumn{2}{|c|}{$\begin{array}{c}\text { Department } \\
\text { of Coaching } \\
\text { Training }\end{array}$} & \multicolumn{2}{|c|}{$\begin{array}{l}\text { Department } \\
\text { of Sports } \\
\text { Management }\end{array}$} & \multicolumn{2}{|c|}{$\begin{array}{c}\text { Department } \\
\text { of } \\
\text { Recreation }\end{array}$} & \multirow{2}{*}{$\begin{array}{c}\begin{array}{c}\text { Pearson } \\
\text { Chi- } \\
\text { Square }\end{array} \\
p\end{array}$} \\
\hline & & $\mathbf{n}$ & $\%$ & $\mathbf{n}$ & $\%$ & $\mathbf{n}$ & $\%$ & $\mathbf{n}$ & $\%$ & \\
\hline \multirow{3}{*}{$\begin{array}{l}\text { I think the practical } \\
\text { courses lectured in } \\
\text { distance education } \\
\text { process will create a } \\
\text { deficiency and } \\
\text { negativity in my } \\
\text { education life in the } \\
\text { next semester or } \\
\text { normal life. }\end{array}$} & I Agree & 129 & 69,0 & 79 & 71,2 & 88 & 65,2 & 14 & 70,0 & \multirow{3}{*}{,404 } \\
\hline & $\begin{array}{l}\text { Neither } \\
\text { Agree } \\
\text { nor } \\
\text { Disagree } \\
\end{array}$ & 28 & 15,0 & 18 & 16,2 & 31 & 23,0 & 5 & 25,0 & \\
\hline & $\begin{array}{l}\text { I } \\
\text { Disagree }\end{array}$ & 30 & 16,0 & 14 & 12,6 & 16 & 11,9 & 1 & 5,0 & \\
\hline \multirow{3}{*}{$\begin{array}{l}\text { I do not consider the } \\
\text { opportunities offered } \\
\text { by our school for } \\
\text { practical courses in } \\
\text { distance education } \\
\text { process as efficacious. }\end{array}$} & I Agree & 109 & 58,3 & 61 & 55,0 & 68 & 50,4 & 11 & 55,0 & \multirow{3}{*}{,467 } \\
\hline & $\begin{array}{l}\text { Neither } \\
\text { Agree } \\
\text { nor } \\
\text { Disagree }\end{array}$ & 45 & 24,1 & 37 & 33,3 & 45 & 33,3 & 6 & 30,0 & \\
\hline & $\begin{array}{l}\text { I } \\
\text { Disagree }\end{array}$ & 33 & 17,6 & 13 & 11,7 & 22 & 16,3 & 3 & 15,0 & \\
\hline \multirow{3}{*}{$\begin{array}{l}\text { I do not think the } \\
\text { education before the } \\
\text { pandemic period and } \\
\text { the education during } \\
\text { the distance education } \\
\text { period have created a } \\
\text { negative difference to } \\
\text { my learning level. }\end{array}$} & I Agree & 65 & 34,8 & 40 & 36,0 & 51 & 37,8 & 7 & 35,0 & \multirow{3}{*}{,882 } \\
\hline & $\begin{array}{l}\text { Neither } \\
\text { Agree } \\
\text { nor } \\
\text { Disagree }\end{array}$ & 56 & 29,9 & 32 & 28,8 & 44 & 32,6 & 8 & 40,0 & \\
\hline & $\begin{array}{l}\text { I } \\
\text { Disagree }\end{array}$ & 66 & 35,3 & 39 & 35,1 & 40 & 29,6 & 5 & 25,0 & \\
\hline \multirow{3}{*}{$\begin{array}{l}\text { I do not think the } \\
\text { courses lectured in } \\
\text { distance education for } \\
\text { the Faculty of Sport } \\
\text { Sciences students } \\
\text { during the pandemic } \\
\text { period are suitable for } \\
\text { our department. }\end{array}$} & I Agree & 114 & 61,0 & 74 & 66,7 & 92 & 68,1 & 16 & 80,0 & \multirow{3}{*}{,368 } \\
\hline & $\begin{array}{l}\text { Neither } \\
\text { Agree } \\
\text { nor } \\
\text { Disagree }\end{array}$ & 44 & 23,5 & 24 & 21,6 & 21 & 15,6 & 3 & 15,0 & \\
\hline & $\begin{array}{l}\text { I } \\
\text { Disagree }\end{array}$ & 29 & 15,5 & 13 & 11,7 & 22 & 16,3 & 1 & 5,0 & \\
\hline \multirow{3}{*}{$\begin{array}{l}\text { I do not think the } \\
\text { theoretical expression } \\
\text { of my practical } \\
\text { courses in distance } \\
\text { education process } \\
\text { contributes upon my } \\
\text { learning level. }\end{array}$} & I Agree & 114 & 61,0 & 71 & 64,0 & 86 & 63,7 & 15 & 75,0 & \multirow{3}{*}{ 665 } \\
\hline & $\begin{array}{l}\text { Neither } \\
\text { Agree } \\
\text { nor } \\
\text { Disagree }\end{array}$ & 43 & 23,0 & 26 & 23,4 & 25 & 18,5 & 4 & 20,0 & \\
\hline & $\begin{array}{l}\text { I } \\
\text { Disagree }\end{array}$ & 30 & 16,0 & 14 & 12,6 & 24 & 17,8 & 1 & 5,0 & \\
\hline \multirow{3}{*}{$\begin{array}{l}\text { I would like the } \\
\text { measurement and } \\
\text { evaluation of my } \\
\text { practical courses to be } \\
\text { fulfilled online after } \\
\text { the pandemic period } \\
\text { is over. }\end{array}$} & I Agree & 57 & 30,5 & 34 & 30,6 & 47 & 34,8 & 4 & 20,0 & \multirow{3}{*}{,588 } \\
\hline & $\begin{array}{l}\text { Neither } \\
\text { Agree } \\
\text { nor } \\
\text { Disagree }\end{array}$ & 56 & 29,9 & 40 & 36,0 & 38 & 28,1 & 9 & 45,0 & \\
\hline & $\begin{array}{l}\text { I } \\
\text { Disagree }\end{array}$ & 74 & 39,6 & 37 & 33,3 & 50 & 37,0 & 7 & 35,0 & \\
\hline
\end{tabular}


THE PRACTICAL COURSES DURING THE PANDEMIC PERIOD

\begin{tabular}{|c|c|c|c|c|c|c|c|c|c|c|}
\hline \multirow{3}{*}{$\begin{array}{l}\text { I would like my } \\
\text { practical courses to be } \\
\text { lectured by visual or } \\
\text { live presentations } \\
\text { instead of theoretical } \\
\text { expression in distance } \\
\text { education process. }\end{array}$} & I Agree & 128 & 68,4 & 72 & 64,9 & 95 & 70,4 & 16 & 80,0 & \multirow{3}{*}{,503 } \\
\hline & $\begin{array}{l}\text { Neither } \\
\text { Agree } \\
\text { nor } \\
\text { Disagree } \\
\end{array}$ & 36 & 19,3 & 28 & 25,2 & 29 & 21,5 & 4 & 20,0 & \\
\hline & $\begin{array}{l}\text { I } \\
\text { Disagree }\end{array}$ & 23 & 12,3 & 11 & 9,9 & 11 & 8,1 & 0 & & \\
\hline \multirow{3}{*}{$\begin{array}{l}\text { I would like to take } \\
\text { my practical courses } \\
\text { face to face if there } \\
\text { were opportunities } \\
\text { during the pandemic } \\
\text { period. }\end{array}$} & I Agree & 144 & 77,0 & 74 & 66,7 & 106 & 78,5 & 15 & 75,0 & \multirow{3}{*}{,395 } \\
\hline & $\begin{array}{l}\text { Neither } \\
\text { Agree } \\
\text { nor } \\
\text { Disagree }\end{array}$ & 27 & 14,4 & 24 & 21,6 & 17 & 12,6 & 4 & 20,0 & \\
\hline & $\begin{array}{l}\text { I } \\
\text { Disagree }\end{array}$ & 16 & 8,6 & 13 & 11,7 & 12 & 8,9 & 1 & 5,0 & \\
\hline \multirow{3}{*}{$\begin{array}{l}\text { I do not believe that I } \\
\text { will be able to put my } \\
\text { theoretical practical } \\
\text { courses into practice } \\
\text { in distance education. }\end{array}$} & I Agree & 80 & 42,8 & 55 & 49,5 & 73 & 54,1 & 12 & 60,0 & \multirow{3}{*}{,225 } \\
\hline & $\begin{array}{l}\text { Neither } \\
\text { Agree } \\
\text { nor } \\
\text { Disagree } \\
\end{array}$ & 81 & 43,3 & 38 & 34,2 & 41 & 30,4 & 7 & 35,0 & \\
\hline & $\begin{array}{l}\text { I } \\
\text { Disagree }\end{array}$ & 26 & 13,9 & 18 & 16,2 & 21 & 15,6 & 1 & 5,0 & \\
\hline \multirow{3}{*}{$\begin{array}{l}\text { I do not consider my } \\
\text { learning level in } \\
\text { practical courses } \\
\text { adequate in distance } \\
\text { education process. }\end{array}$} & I Agree & 116 & 62,0 & 71 & 64,0 & 94 & 69,6 & 14 & 70,0 & \multirow{3}{*}{,323 } \\
\hline & $\begin{array}{l}\text { Neither } \\
\text { Agree } \\
\text { nor } \\
\text { Disagree }\end{array}$ & 45 & 24,1 & 30 & 27,0 & 21 & 15,6 & 4 & 20,0 & \\
\hline & $\begin{array}{l}\text { I } \\
\text { Disagree }\end{array}$ & 26 & 13,9 & 10 & 9,0 & 20 & 14,8 & 2 & 10,0 & \\
\hline
\end{tabular}

When the attitude levels of the students participating in the study towards the practical courses lectured in distance education were analyzed, it was found that there was no statistically significant difference in all items $(p>0,05)$.

\section{Discussion and Conclusion}

COVID-19 which started in Wuhan, China in November 2019 and was declared as a pandemic by the World Health Organization in March 2020 affected the education sector as well as many other ones. With the spread of the pandemic, education was suspended in our country and the distance education model was decided to be administered in all educational institutions since the spread of the pandemic could not be stopped. In this context, it was observed that the majority of educational institutions were blindsided for transition to distance education (Şen and Kizilcalioğlu, 2020). Since theoretical and computer-based practice courses were carried out in distance education model until today, there appeared greater difficulties in these courses due to the first trial of practice courses lectured in laboratory and workshop environments.

It was very difficult to reach concrete data due to the fact that the rate of practical courses was high in the Faculty of Sport Sciences and there was almost no research carried out on how the distance education model administered during the pandemic 
process could affect the learning levels of the students. In this context, this research was a completely original study, and would provide remarkable contributions upon further studies on this subject. Furthermore, since the literature on the subject was very limited, the comparisons were made with the studies in similar quality. The results of this study which was carried out depending on the importance of the process were as follows.

In the questionnaire we performed, the rate of students who answered yes to the question "Would you like distance education process to continue?" was (n=116, 25.1\%), and the rate of students who answered no was $(n=346,74.9 \%)$. When considering the values obtained, it was noticed that the number of students who did not want the distance education process to continue was quite high. In this context, the students of the Faculty of Sports Sciences had a high desire level to have face-to-face education. It was considered that some of the students answered no thinking that it was easier to pass the courses in distance education process.

In comparison of the questionnaire performed to measure the attitudes of students towards the practical courses lectured in distance education according to the variable of grade, a significant difference $(p>0,05)$ was determined between the grades when the answers given to the items of "I would like to take my practical courses face to face if there were opportunities during the pandemic period" and "I do not believe that I will be able to put my theoretical practical courses into practice in distance education" were analyzed. No significant difference was determined in other items. When the frequency values were analyzed, it was noticed that students' willingness to take practical courses face to face was high, but the willingness rate of the fourth-grade students was lower than the other grades. It was considered that this could be arisen due the fourth-grade students' regarding themselves as trained and competent, especially in terms of practical courses, since they fulfilled all their theoretical and practical training face to face before the pandemic period. In the item of "I do not believe that I will be able to put my theoretical practical courses into practice in distance education" in which we found a significant difference between the grades, the frequency values of the fourth-grade students were specified to be high. It was noticed that the data obtained had a quality supporting the previous item. In this sense, it was noticed that fourth grade students believed they could put theoretically lectured practical courses into practice depending on the acquisitions they had in their face-to-face education in previous three-year period up to the pandemic process.

Kahraman stated that in the process experienced with COVID-19 pandemic, all theoretical and practical courses were offered to the students with distance education method, and distance education model was administered compulsorily since formal education was completely stopped. Furthermore, he stated that distance education system was beneficial and sufficient for theoretical lessons, but in fields where some part of the curriculum was administered practically, difficulties in practical course were experienced in distance education model, and therefore the practical courses could not be maintained with distance education method (Kahraman, 2020). Kürtüncü and Kurt suggested that the practical courses lectured in the COVID-19 pandemic were inefficient 
and these courses should be repeated in an accelerated manner in following periods (Kürtüncü and Kurt, 2020). Demir stated in his study on distance education from the perspectives of students that because students constantly found themselves in a listening position in a virtual classroom environment, they felt themselves isolated. Furthermore, it was stated that technological inadequacies were possible to harm the flow of information and such situations could be efficient upon student motivation (Demir, 2014). Şen and Kızılcalığlu reported that disadvantages of distance education are felt more especially in practical courses (Şen and Kızılcalığlu, 2020).

Yolcu stated in his study on distance education experiences of preservice classroom teachers during the Coronavirus (Covid-19) pandemic process that the vast majority of students (83\%) experienced the fear of their education to remain incomplete (Yolcu, 2020). In the study carried out on conservatory students' perceptions and experiences related to distance education during the Covid-19 outbreak, Umuztaş and Taş stated that students generally experienced performance loss in practical areas (Instrument and Practices) (Umuztaş \& Taş, 2020). In a study, Kırbaş mentioned that the faculty members carrying on their duties in the Faculties of Sports Sciences in universities considered lecturing practical courses with distance education was inadequate and inefficient (Kurbaş, 2020). Current literature information indicated that the theoretical lectured practical courses lectured in both sports sciences and other branches during the pandemic process had a negative effect upon the education level of the students, and this supported the data obtained in our study.

In the study, the attitudes of Faculty of Sports Sciences students towards the practical courses lectured in distance education process were analyzed in terms of the variable of department, as well. According to the data obtained, it was determined that there was no statistically significant difference between the departments in all asked items $(p>0,05)$. In this sense, it was considered that the attitudes of the students of the Department of Physical Education and Sports Teaching, Department of Coaching Education, Department of Sports Management and Department of Recreation were the same towards the practical courses lectured in distance education.

In conclusion, it was considered that the practical courses lectured in distance education in the Faculty of Sports Sciences negatively affected the learning levels of the students during the pandemic caused by the Covid-19 virus. It was observed that most of the students believed they would not be able to put the theoretical courses lectured in distance education into practice during this period and this would have a negative effect on their development. Furthermore, in this process, insofar as circumstances permit, it was considered that lecturing the courses face-to-face was adopted by the students, and there was a consensus on this.

\section{Conflict of Interest Statement}

The authors declare no conflicts of interests. 


\begin{abstract}
About the Authors
Mustafa Kaya, Sivas Cumhuriyet University, Faculty of Sport Sciences, Coaching Training Department. His area of specialization is physical activity, performance enhancement.
\end{abstract}

Alperen Çırçır, Sivas Cumhuriyet University, Graduate Student of Health Sciences Institute.

Alper Eyinç, Sivas Cumhuriyet University, Yıldızeli Vocational School. His specialization is educational sciences.

Mehmet Kargün, Tokat Gaziosmanpaşa University, Sport Sciences, Sports Management Department. His specialty is sports management.

Recep Bozdemir, Tokat Gaziosmanpaşa University, Sport Sciences, Coaching Training Department. His specialty is physical education and sports teaching.

\title{
References
}

Afşar, B. ve Büyükdoğan, B. (2020). Assessments of FBAS and FSHS Students About Distance Education During the Covid-19 Pandemic Period, Karatay Journal of Social Research, October (5), 161-182.

Aktaş Ö., Büyüktaş B., Gülle M., Yıldız M. (2020). Sports Science Students' Attitudes Towards Distance Education During Isolation Days Caused by Covid-19 Virus, Sivas Cumhuriyet University Journal of Sport Sciences Volume 1, Issue 1.

Baştürk, S. Taştepe, M. (2013). Universe and Sample. Scientific Research Methods, Ankara: Vize Publishing, 129-159.

Büyüköztürk, S., Çakmak, E., Akgün, Ö.E., Karadeniz, S., \& Demirel, F. (2012). Scientific Research Methods (18th edition). Ankara: Pegem Academy Publishing.

Çelik F. Yenal T. H. (2020). Covid-19 and Still Life. Mediterranean Journal of Sport Sciences, Volume 3, Issue 2

Demir, E. (2014). An Overview of Distance Education, Dumlupinar University Journal of Social Science, Vol: 39, Pages 203-212

Dong E. Du H. Gardner L. (2020). An interactive web-based dashboard to track COVID19 in real time. Lancet Infect Dis, (20)30120-1

Jiménez-Pavón D. Carbonell-Baeza A. Lavie CJ. (2020). Physical exercise as therapy to fight against the mental and physical consequences of COVID-19 quarantine. Special focus in older people (published online ahead of print, Mar 24). Prog Cardiovasc Dis, S0033-0620 (20)30063-3.

Kahraman M. E. (2020). The Effect of COVID-19 Epidemic on Applied Courses and the Implementation of These Courses by Distance Education: Example of Basic Design Course, Civilization Art- Journal of IMU Art Design and Architecture Faculty, Volume: 6, Issue: 1, p. 44-56 
Kırbaş Ş., (2020). The Views of Physical Education and Sports Teaching Instructors on Education in the COVID-19 Period, Journal of Education and Learning; Vol. 9, No. $6 ; 2020$

Kürtüncü, M. Kurt, A. (2020). Problems Of Nursing Students in Distance Education in the Covid-19 Pandemic Period, Eurasian Journal of Researches in Social and Economics (Ejrse), Volume 7 Issue 5, S 66-77

Li Q. Guan X. Wu P. Wang X. Zhou L. Tong Y. (2020). Early transmission Dynamics in Wuhan, China, of novel coronavirus-infected pneumonia. N Engl J Med. Jan. doi: 10.1056/NEJMoa2001316.

Palacios, M. Cruz, M. Santos, E. M. A. Velázquez Cervantes, M A. León Juárez, M. (2021). COVID-19, a Worldwide Public Health Emergency. Revista Clínica Española, Rev Clin Esp. 221:55 - 61.

Şen, Ö. Kızılcalığlu, G. (2020). Determining The Views of University Students and Academics on Distance Education During the Covid-19 Pandemic, International Journal Of 3d Printing Technologies and Digital Industry 4:3 (2020) 239-252

Tuncer, M. Bahadir, F. (2017). Evaluation of the Distance Education Programs according to Student Views that Learned in These Programs. Journal of Educational Reflections, 1(2), 27-36.

Umuztaş, S. Baş, A. H. (2020). Examining The Perceptions and Experiences of Conservatory Students Regarding Distance Education During the Covid-19 Pandemic (Tokat City Example), Yegâh Mûsikî Journal, Volume III Issue 2, pp.204-220

Yolcu, H. H. (2020). Preservice elementary teachers' distance education experiences at the time of coronavirus (covid-19) pandemic, Journal of Open Education Applications and Research, Volume 6, Issue 4, 237-250

Council of Higher Education, "Press Release" https://covid19.yok.gov.tr/Documents/alinan-kararlar/03-distance-educationrelated-taken-decision.pdf. Nov 20, 2020. 
Mustafa Kaya, Alperen Çırçır, Alper Eyinç, Mehmet Kargün, Recep Bozdemir

Creative Commons licensing terms

Authors will retain the copyright of their published articles agreeing that a Creative Commons Attribution 4.0 International License (CC BY 4.0) terms will be applied to their work. Under the terms of this license, no permission is required from the author(s) or publisher for members of the community to copy, distribute, transmit or adapt the article content, providing a proper, prominent and unambiguous attribution to the authors in a manner that makes clear that the materials are being reused under permission of a Creative Commons License. Views, opinions and conclusions expressed in this research article are views, opinions and conclusions of the author(s). Open Access Publishing Group and European Journal of Physical Education and Sport Science shall not be responsible or answerable for any loss, damage or liability caused in relation to/arising out of conflict of interests, copyright violations and inappropriate or inaccurate use of any kind content related or integrated on the research work. All the published works are meeting the Open Access Publishing requirements and can be freely accessed, shared, modified, distributed and used in educational, commercial and non-commercial purposes under a Creative Commons attribution 4.0 International License (CC BY 4.0). 